\title{
La importancia del color: morfos florales, tasas de visita y éxito reproductivo en el arbusto Sarothamnus scoparius
}

\author{
Sabrina S. Gavini ${ }^{1} \&$ Alejandro G. FarJi-Brenter ${ }^{1,2, \bigotimes}$ \\ ${ }^{1}$ Centro Regional Universitario Bariloche, Universidad Nacional del Comahue. ${ }^{2}$ Laboratorio Ecotono, INIBIOMA-CONICET.
}

\begin{abstract}
Resumen. El estudio de la variación intraespecífica del color de las flores nos permite comprender mejor el papel de los polinizadores como agentes de selección, y su efecto sobre la abundancia de las variantes florales. Estudiamos la influencia de la variación en los colores florales sobre la tasa de visita a las flores y la reproducción de Sarothamnus scoparius (retama), un arbusto exótico común en el NO de la Patagonia. En particular, determinamos si la mayor abundancia de arbustos con flores amarillas en relación a los arbustos con flores rojas o naranjas se debía a una tasa de visita diferencial por parte de sus potenciales polinizadores. Para eso, 1) medimos la tasa de visita a flores amarillas, rojas y naranjas en situaciones naturales y en plantas cuyo color de las flores fue manipulado; 2) determinamos si la tasa de visita a las flores rojas y naranjas dependía de su distancia al morfo floral más abundante (amarillo), y 3) estimamos la producción de frutos / flor y semillas/fruto en arbustos con flores amarillas y rojas. Encontramos que: 1) tanto en situaciones naturales como en flores cuyo color fue manipulado, las flores amarillas recibieron entre 1 y 4 veces más visitas que las otras variantes; 2 ) la tasa de visita a las flores rojas y naranjas decrecieron de manera abrupta a medida que aumentaba su distancia a las flores amarillas (7 veces en solo $100 \mathrm{~cm}$ ), y 3) tanto la producción de frutos/flor como la de semillas/fruto fue $\sim 40 \%$ mayor en los arbustos con flores amarillas comparado con aquellos de flores rojas. Estos resultados sugieren que la mayor abundancia de arbustos con flores amarillas se debe a que sus potenciales polinizadores prefieren este color, lo que se traduce en una reproducción mayor de esta variante floral. Este trabajo ilustra la importancia de los polinizadores como eventuales agentes selectivos de las características florales, así como la influencia del vecindario floral en dicho proceso
\end{abstract}

[Palabras clave: Bombus, Patagonia, polinización, vecindario floral]

\begin{abstract}
Aвstract. The importance of color: floral morphs, visit rates and fitness in the shrub Sarothamnus scoparius. The study of the intraspecific variability in the flower color may help us to better understand the role of pollinators as selective agents end their effect on the local abundance of floral morphs. We studied the influence of intraspecific variation of flower color on the floral visitation rate and the fitness of Sarothamnus scoparius, a common exotic shrub in Northern Patagonia. Particularly, we determined whether the higher abundance of shrubs with yellow-flowers compared with those with red or orange flowers was consequence of a differential visitation rate of potential pollinators. We 1) measured the visitation rate to yellow flowers and their variants red and orange in natural conditions and in flowers where their color was manipulated;2) determined whether the visitation rate to red and orange flowers depended on their distance to yellow flowers, and 3) estimated the production of fruit set and seed set in yellow and red flower morphs. We found that: 1) in both, natural and color-modified conditions, yellow flowers received 1-4 times more visits than red or orange flowers; 2 ) the visitation rate to red and orange flowers decreased abruptly as the distance to yellow flowers increases (7 times in only $100 \mathrm{~cm}$ ), and 3) both fruit and seed set were $\sim 40 \%$ higher in shrubs with yellow flowers compared with those with red flowers. These results suggest that the higher abundance of shrubs with yellow flowers is consequence of a higher visitation rate of potential pollinators, which in turn produce a higher fitness in this floral morph. This work illustrates the importance of pollinators modeling flower characteristics and the influence of floral neighborhood in such process.
\end{abstract}

[Keywords: Bombus, Patagonia, pollination, floral neighborhood]

\section{INTRODUCCIÓN}

El polimorfismo de coloración en las flores es un fenómeno común, con implicancias tanto evolutivas como ecológicas. Por un lado, es un modelo conceptual útil para comprender mejor los factores que mantienen la diversidad (Waser \& Price 1981; Wessinger \& Rausher

\section{Editor Asociado: Esteban Jobbágy}

$\triangle$ alefarji@yahoo.com
2012) y para estudiar cómo los agentes selectivos pueden modelar características de los organismos (Galen 1989; Levin \& Brack 1995; Renoult et al. 2014). Por otra parte, diferentes colores pueden variar en su nivel de atracción para los visitantes florales, lo que afecta no sólo las tasas de reproducción de las plantas sino también al ensamble 
de polinizadores y redes de interacciones asociadas (Eckhart et al. 2006). Por todas estas razones, el estudio de la variación del color en flores es un tópico de interés actual.

A menudo, las variaciones florales han sido explicadas por selección mediada por polinización: diferentes tipos de polinizadores pueden actuar de agentes selectivos a favor o en contra de distintos caracteres florales, incluyendo el color (Weiss 1995; Renoult et al. 2014). Los polinizadores tienen la capacidad de detectar cambios sutiles en los colores y de reducir su tasa de visita a aquellas variaciones menos atractivas (Papiorek et al. 2013; Land \& Chittka 2013). Esta respuesta a variaciones cromáticas en las flores también es común dentro de una misma especie. Por ejemplo, Mimulus aurantiacus posee dos morfos florales, rojo y amarillo, los que atraen a diferentes especies de polinizadores (Streisfeld \& Kohn 2007). Sin embargo, existen otros factores que también pueden explicar el origen y/o el mantenimiento de las variaciones intraespecíficas en los colores florales, como cambios en el microclima o condiciones edáficas (Arista et al. 2013). En consecuencia, el papel de los polinizadores en el mantenimiento de las variaciones del color floral dentro de una misma especie es aún objeto de controversia y de estudio (Strauss \& Whittall 2006).

El arbusto exótico Sarothamnus scoparius (de aquí en más, retama) representa un buen modelo para estudiar la importancia de los polinizadores en el mantenimiento de variaciones intraespecíficas en los colores florales. Primero, es un arbusto común en el NO de la Patagonia, es sencillo de ubicar y posee flores fáciles de observar y manipular. Segundo, presenta variaciones contrastantes en el color de sus flores, con mayor abundancia de individuos con flores completamente amarillas y menor de aquellos con flores parcialmente naranjas o rojas. Tercero, su polen es liberado de manera explosiva a los visitantes florales sólo cuando éstos visitan la flor y ocasionan la apertura de sus pétalos-quilla; por esta razón es sencillo saber si una flor fue visitada o no. Finalmente, sus polinizadores principales son abejorros (Apidae: Bombini), que responden de forma diferencial a estas variaciones en colores (Chittka \& Menzel 1992; Skorupski \& Chittka 2010).

En este estudio empleamos las variaciones de colores florales de la retama para analizar la influencia del color sobre la tasa de visita de los polinizadores, y su eventual efecto sobre la reproducción. Nuestra hipótesis es que la mayor abundancia natural de plantas con flores completamente amarillas se debe a una mayor preferencia de ese color por los polinizadores, lo que se traduce en una producción mayor de frutos y semillas de este morfo con respecto a los rojos y naranjas. En consecuencia, esperamos que: a) las flores amarillas reciban una mayor tasa de visita que las rojas o naranjas; b) que la tasa de visita a las flores rojas o naranjas dependa de su vecindario floral (es decir, predecimos una relación negativa entre la tasa de visita a las flores rojas o naranjas y su distancia a las flores amarillas), y c) que las plantas con flores amarillas presenten una mayor relación de frutos/flor y semillas / fruto que plantas con flores rojas o naranjas. Para ello realizamos observaciones en condiciones naturales de la tasa de visita a las diferentes variantes florales y medimos su tasa de producción de frutos y semillas; por último analizamos como cambios experimentales en el color de las flores afectaba las tasas de visita de los polinizadores.

\section{MATERIALES Y MÉTODOS}

\section{Sitio y especie de estudio}

El trabajo se realizó en la ciudad de San Carlos de Bariloche, Argentina, y sus alrededores $\left(41^{\circ} 8^{\prime} \mathrm{S}\right.$, $71^{\circ} 19^{\prime} \mathrm{O}$ ), en diferentes parches de bosque y matorral con gran abundancia de retama. La precipitación anual es de $\sim 1800 \mathrm{~mm}$ y la temperatura promedio varía entre $2{ }^{\circ} \mathrm{C}$ en invierno hasta $13^{\circ} \mathrm{C}$ en verano. La retama (Sarothamnus scoparius, Fabaceae) es una especie arbustiva exótica originaria de Europa, hoy ampliamente distribuida en el NO de la Patagonia. Sus flores son pentámeras, con un pétalo posterior en forma de estandarte (el mayor), dos pétalos laterales (o alas) y dos anteriores unidos formando la quilla. Las flores son de color amarillo brillante. Sin embargo, algunas plantas presentan morfos florales en los que los dos pétalos laterales pueden ser de color rojo o naranja (Figura 1). Como nunca un mismo arbusto presenta más de una variante floral, cuando hablemos de flores amarillas, rojas o naranjas estaremos refiriéndonos a arbustos con ese morfo floral. El mecanismo de dehiscencia del polen es explosivo: los estambres y el pistilo están sometidos a una gran tensión bajo la quilla y son expulsados cuando el polinizador ejerce presión. Cuando el potencial polinizador visita las flores, sus movimientos y zumbidos estimulan la apertura explosiva de los pétalos-quilla; esto libera los estambres y el pistilo, de tal forma que la parte ventral del animal queda impregnada de polen (Muller 1883). Los polinizadores más comunes en NO de la Patagonia son las abejas domésticas (Apis mellifera) y, en especial, los abejorros (Bombus spp.). 
Por lo general, estos visitantes florales permanecen poco tiempo en un parche (entre 3 y 5 minutos) y provocan la apertura de, eventualmente, entre 15 y 20 flores de un mismo arbusto o de ramas de arbustos cercanos antes de emprender vuelo (S. Gavini, observación personal). Por esta razón, se supone que el intercambio de polen entre flores de una misma planta es proporcionalmente bajo (una arbusto mediano puede tener miles de flores). Debido al mecanismo explosivo de polen descripto más arriba, cuando la flor está cerrada implica que aún no ha sido visitada, mientras que cuando está abierta con los estambres y el pistilo expuestos, implica que ha sido visitada. Su sistema reproductivo es básicamente autoincompatible, y la polinización cruzada incrementa varias veces su producción de semillas, por lo cual se estima que esta especie se encuentra limitada por polen (Rodríguez-Riaño et al. 1999). El fruto es una cápsula en forma de legumbre, con dehiscencia también explosiva.

\section{Metodología}

Para estimar la abundancia relativa de los diferentes morfos florales, realizamos observaciones en 5 sitios con presencia del arbusto ubicados a lo largo de $\sim 50 \mathrm{~km}$ sobre la Ruta Nacional 40, desde la entrada hasta el final del municipio de Bariloche. En cada sitio ubicamos al azar 3 parcelas de 10x10 $\mathrm{m}$, en donde contamos todos los individuos de cada morfo.

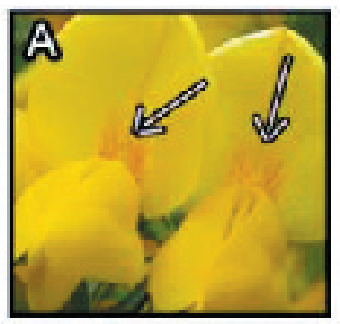

Figura 1. Variedades (morfos) florales del arbusto Sarothamnus scoparius (retama). Variedad amarilla (A), la más frecuente, $\mathrm{y}$ variedades roja (B) y naranja (C), menos frecuentes. Las flechas señalan el patrón de guías de néctar debajo del pétalo principal.

Figure 1. Floral morphs of the shrub Sarothamnus scoparius (retama). Yellow morph (A), the more abundant, and red (B) and orange $(C)$ morphs, less abundant. The arrows indicate the pattern of nectar guide in the main petal.

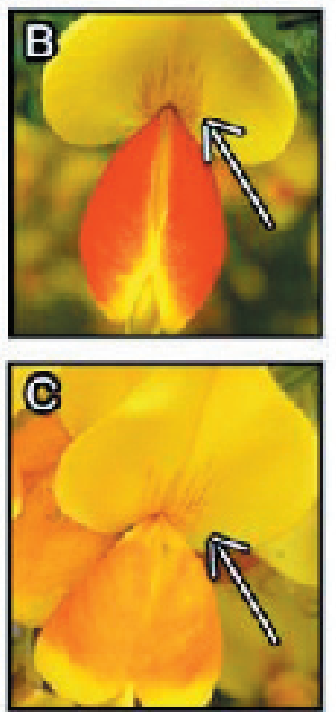

Tasas de visita de polinizadores y morfos florales: mediciones comparativas y correlativas

Para analizar el efecto del color sobre la tasa de visita de potenciales polinizadores trabajamos en un gran parche ubicado en las cercanías de la costa del lago Nahuel Huapi, a $8 \mathrm{~km}$ de la ciudad de Bariloche, en donde están presentes las 3 variantes florales. Elegimos al azar ramas con flores cerradas (i.e., no visitadas) y en ellas marcamos 25 flores. Marcamos un total de 210 ramas de los tres morfos florales (65 con flores amarillas, 60 ramas con flores naranjas y 85 ramas con flores rojas). Todas las ramas se ubicaban a alturas similares desde suelo (entre 1 y $1.5 \mathrm{~m}$ ), estaban libremente expuestas a potenciales visitas (i.e., no estaban ocultas tras la vegetación) y pertenecían a diferentes plantas. Las ramas con flores de diferente color se encontraban relativamente entremezcladas en el espacio (aunque, como siempre, las flores amarillas eran mayoría; ver Resultados). Cada flor fue marcada individualmente. Luego de una semana, volvimos a visitar el sitio de estudio y contabilizamos, para cada rama, cuantas de las 25 flores marcadas pasaron de cerradas a abiertas (i.e., fueron visitadas). La ocurrencia de visita se expresó en porcentaje para cada morfo floral como:

\section{(Nro. de flores abiertas por rama $/ 25)^{*} 100$} (Ecuación 1)

Para determinar la influencia de la cercanía de flores amarillas (el morfo más abundante) sobre la visita de potenciales polinizadores a las flores de otros colores, medimos la tasa de visita en las flores rojas y naranjas marcadas como fuera explicado anteriormente, y su distancia a la rama más cercana con flores amarillas. La tasa de visita entre morfos florales (\%) fue analizada mediante un análisis de varianza de una vía, considerando al color como factor fijo. Las diferencias entre los niveles del tratamiento (i.e., colores) fueron analizadas "a posteriori" con una prueba de Tukey. El efecto de la distancia al morfo amarillo sobre la tasa de visita a los morfos rojo y naranja fue analizado mediante regresiones simples.

\section{Tasas de visita de polinizadores y morfos florales: mediciones experimentales}

Para determinar de manera experimental el efecto del color de las flores sobre la ocurrencia de visita de potenciales polinizadores manipulamos los colores de las flores amarillas y rojas, y cuantificamos el número de flores visitadas después de la manipulación. Seleccionamos al azar 24 ramas con flores amarillas y 24 ramas con flores rojas, cada rama con 25 flores, pertenecientes a diferentes plantas. No realizamos experimentos con flores naranjas. Cada rama fue asignada al azar a un tratamiento dentro de cada color de flor, en donde a) se dejaban las 25 flores con el color original (control), b) se pintaban las 25 flores con 
el otro color (tratamiento), y c) se pintaban a las 25 flores con su mismo color (control por el efecto de la pintura). En consecuencia, realizamos los siguientes 6 tratamientos: AC (flores amarillas sin manipulación, control), APA (flores amarillas pintadas de amarillo), APR (flores originalmente amarillas pintadas de rojo), RC (flores rojas sin manipulación, control), RPR (flores originalmente rojas pintadas de rojo) y RPA (flores originalmente rojas pintadas de amarillo). Cada tratamiento consistía entonces de 8 ramas, con 25 flores cada rama en las que todas las flores eran sometidas al mismo tratamiento. Para reducir el efecto del vecindario floral removimos todas las flores de otras ramas a una distancia aproximada de $1 \mathrm{~m}$ radio alrededor de cada rama sometida a nuestros tratamientos. Luego de establecer los tratamientos, las ramas fueron visitadas a la semana siguiente. La ocurrencia de visita fue calculada de igual manera que en las mediciones anteriores, usando la Ecuación 1. En total observamos 1200 flores (8 ramas por 6 tratamientos, 25 flores/rama). La ocurrencia de visita fue analizada mediante un análisis de varianza de una vía, en el que los tratamientos fueron considerados como factor fijo. Las diferencias entre los niveles del tratamiento fueron analizadas "a posteriori" por medio de una prueba de Tukey.

\section{Adecuación y morfos florales: estimación de frutos y semillas}

Para establecer si los diferentes morfos florales variaban en su adecuación, estimamos la relación de frutos/flor ("fruit set") y el número de semillas / fruto (o sea, semillas viables/semillas total o "seed set") en individuos con morfos florales amarillos y rojos. Para ello, durante la primavera 2014 contamos el número de flores en $\sim 50 \mathrm{~cm}$ de rama, en dos ramas de 6 arbustos por morfo floral (i.e., 12 ramas por morfo). Dichas flores fueron marcadas y revisadas hacia el final de la época de fructificación, momento en el que cuantificamos el número de frutos producidos/flores marcadas. Este parámetro varía entre 0 (ningún fruto producido) y 1 (todas las flores fueron exitosamente polinizadas y se convirtieron en fruto). Para estimar la relación de semillas/fruto, elegimos 15 frutos al azar por rama y en ellas contabilizamos el número de semillas viables/totales (viables+abortadas). Este valor también oscila entre 0 (ninguna semilla viable por fruto) y 1 (todas las semillas del fruto son viables). Las estimaciones de frutos/flor y semillas/fruto entre los morfos amarillos y rojos fueron analizadas mediante análisis de varianzas anidados para cada variable respuesta; en ambos casos las ramas fueron anidadas a los árboles.

\section{REsUlTADOS}

El morfo amarillo resultó mucho más abundante que los morfos rojo y/o naranja, lo cual confirma las observaciones a simple vista. En términos generales, de los parches observados, $75 \%$ de la cobertura presentaba flores amarillas, mientras que $25 \%$ exhibía flores de los morfos rojos y/o naranjas.

\section{Tasas de visita de polinizadores y morfos florales: mediciones comparativas y correlativas}

Las observaciones a campo nos permitieron confirmar que la apertura de las flores era realizada de forma activa por los visitantes florales, y que éstos no solían visitar las flores ya abiertas. En consecuencia, la cantidad relativa de flores abiertas en una rama fue considera como un estimador confiable de la tasa de visita de los potenciales polinizadores a los diferentes morfos florales. La tasa de visita de los polinizadores varió entre los morfos florales: fue mayor en las flores amarillas que en las flores rojas y naranjas, las cuales presentaron tasa de visita similares entre sí $\left(F_{2207}=61.3, P<0.001\right.$ y prueba a posteriori de Tukey, Figura 2). Mientras que en el período de muestreo las ramas con flores amarillas presentaron $55 \pm 2 \%$ de flores visitadas (media $\pm E S$ ), las flores rojas y naranjas mostraron una tasa de visita de $25 \pm 3 \%$ y $23 \pm 2 \%$, respectivamente. Además, la cercanía a flores amarillas incrementó la tasa de visita a flores de los otros colores. Cuando las flores naranjas o rojas se ubicaban a $\sim 50 \mathrm{~cm}$ de distancia de las flores amarillas, su tasa de visita se reducía $\sim 60 \%$, mientras que cuando estaban a 100-150 cm su tasa de visita bajaba a $~ 10 \%$ (Figura 3).

\section{Visitas de polinizadores y morfos florales: mediciones experimentales}

Los resultados experimentales fueron consistentes con lo observado en el campo. Más allá de si el color era natural o pintado, las flores amarillas presentaron una mayor tasa de visita que las flores rojas $\left(F_{5,42}=53.5\right.$, $P<0.001$; Figura 4). Dentro de cada color también se notó un leve efecto de la pintura, que redujo la tasa de visita respecto al color natural, pero este potencial sesgo de la manipulación no influyó sobre el patrón general de una mayor visita al morfo amarillo. Mientras las flores amarillas presentaron una tasa de visita de $\sim 47 \%$ [AC $=55.5 \pm 4 \%$; APA $45 \pm 3 \%$; RPA $40.5 \pm 3 \%$ (media $\pm E S$ )], las flores rojas presentaron una tasa de visita de $\sim 8 \%[\mathrm{RC}=17 \pm 4 \%, \mathrm{RPR}=5.5 \pm 2 \%, \mathrm{APR}=2 \pm 1 \%$ (media \pm ES); Figura 4]. 


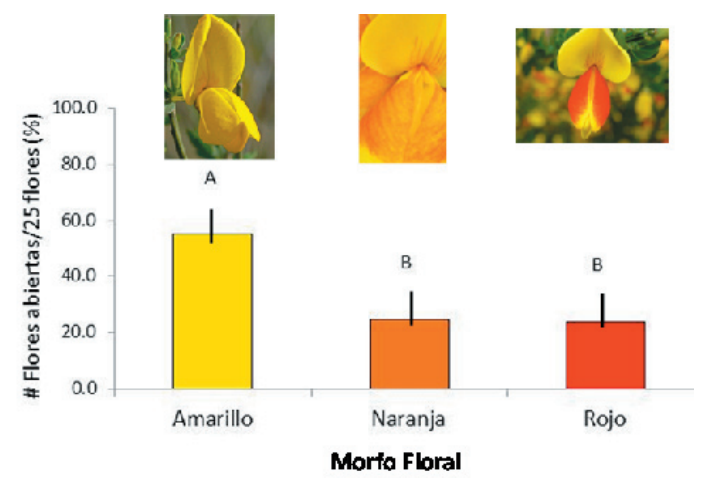

Figura 2. Tasa media de visita $( \pm \mathrm{ES})$ ([Nro. de flores abiertas $/ 25$ flores $\left.]^{*} 100\right)$ a los diferentes morfos florales en condiciones naturales. Letras diferentes indican diferencias estadísticamente significativas $(P<0.05$, prueba "a posteriori" de Tukey).

Figure 2. Mean rate of floral visits $( \pm \mathrm{SE}$ ) ([No. opened flowers $/ 25$ flowers $]^{*} 100$ ) to the different floral color morphs in natural conditions. Different superscripts mean statistical significant differences $(P<0.0$ ukey poshoc test).

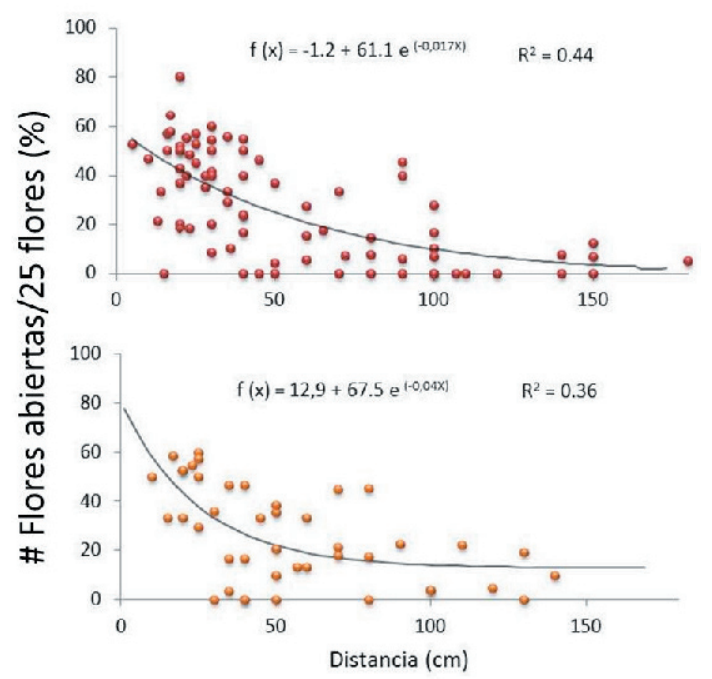

Figura 3. Tasa media de visita $( \pm \mathrm{ES})$ ([Nro. de flores abiertas $/ 25$ flores] ${ }^{*} 100$ ) a flores de mofo rojo (arriba) y naranja (abajo) en relación a su distancia a la rama con flores amarillas más cercana. Se observa una reducción de la tasa de visita a medida que la distancia a ramas con flores amarillas se incrementa.

Figure 3. Mean rate of floral visits $( \pm \mathrm{SE}$ ) ([No. opened flowers / 25 flowers] ${ }^{*} 100$ ) to red (above) and orange (below) flower morphs in relationship with their distance to the nearest branch with yellow flowers. A reduction in the rate of visit is seen as the distance to branches with yellow flowers increases

\section{Adecuación y morfos florales: estimación de frutos y semillas}

Ambas medidas de adecuación dependieron del color de las flores y fueron mayores en las plantas con flores amarillas (Figura 5). La relación de frutos producidos por flor dependió del color del morfo floral $\left(F_{1,11}=9.7\right.$,

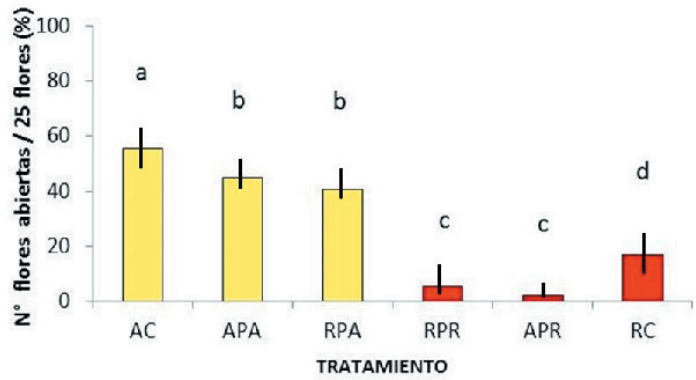

Figura 4. Tasa media de visita $( \pm \mathrm{ES})$ ([Nro. de flores abiertas $/ 25$ flores $]^{*} 100$ ) a flores sometidas a diferentes manipulaciones para modificar su color. $\mathrm{AC}=$ flores originalmente amarillas sin manipular (control), $\mathrm{APA}=$ flores originalmente amarillas pintadas de amarillo (control del efecto de la pintura), RPA=flores originalmente rojas pintadas de amarillo, $\mathrm{RC}=$ flores originalmente rojas sin manipular (control), RPR=flores originalmente rojas pintadas de rojo (control del efecto de la pintura) y $\mathrm{APR}=$ flores originalmente amarillas pintadas de rojo. Los colores de las barras representan el color final de las flores luego de las manipulaciones. Letras diferentes indican diferencias estadísticamente significativas $(P<0.05$, prueba "a posteriori" de Tukey).

Figure 4. Mean rate of floral visits $( \pm \mathrm{SE})([$ No. of opened flowers/25 flowers] ${ }^{*} 100$ ) to flowers under different manipulations that modified their color. AC=flowers originally yellow without manipulation (control), $\mathrm{APA}=$ flowers originally yellow painted yellow (control for painting), RPA=flowers originally red painted yellow, RC=flowers originally red painted red (control), $\mathrm{RPR}=$ flowers originally red painted red (control for painting), and $\mathrm{APR}=$ flowers originally yellow painted red. The bars color represents the final color of the flowers after the manipulations. Different superscripts mean statistical significant differences $(P<0.05$, Tukeypos-hoc test).

$P<0.001)$, pero no de la rama ni del árbol $(0.45<P<0.52$ en ambos casos). El morfo amarillo presentó una mayor cantidad de frutos/flor que el morfo rojo [ $0.96 \pm 0.02$ versus $0.72 \pm 0.07$ respectivamente (media $\pm \mathrm{ES}$ )]. La relación de semillas/fruto también dependió del color de la flor $\left(F_{1,28}=103, P<0.001\right)$, pero no de la rama ni del árbol $(0.34<P<0.47$ en ambos casos). Los frutos provenientes de flores amarillas presentaron una mayor proporción de semillas potencialmente viables que los frutos provenientes de flores rojas $[0.73 \pm 0.01$ versus $0.48 \pm 0.02$, respectivamente (media \pm ES)].

\section{DisCUSIÓN}

El color de las flores afectó la tasa de visita y reproducción del arbusto Sarothamnus scoparius. Los individuos con flores amarillas (morfo más abundante) presentaron entre 2 y 5 veces más visitas que los individuos con flores rojas o naranjas. Además, la tasa de visita a flores rojas o naranjas se redujo hasta 5 veces si éstas se distanciaban a más 
de100 cm de flores amarillas. Finalmente, los individuos con flores amarillas produjeron $30 \%$ más de frutos/flor y $50 \%$ más de semillas potencialmente viables por fruto que los individuos con flores rojas. Estos resultados sugieren que la mayor abundancia de individuos con flores amarillas se debe a que este color de flor es más atractivo que los colores rojo y naranja. Entonces, al recibir más visitas presenta una tasa reproductiva mayor y su abundancia relativa aumenta.

Estos resultados son consistentes con otros trabajos en los que observaciones y manipulaciones en el color de las flores determinaron cambios en las tasas de visita de los polinizadores y en la producción de semillas (Stanton 1987; Meléndez-Ackerman \& Campbell 1998). La mayor atracción por las flores amarillas puede deberse a que las variantes florales rojas y naranjas son menos conspicuas o menos atractivas visualmente para los abejorros. Por lo general, estos insectos no perciben el color rojo y sus variantes cromáticas cercanas (Chittka \&

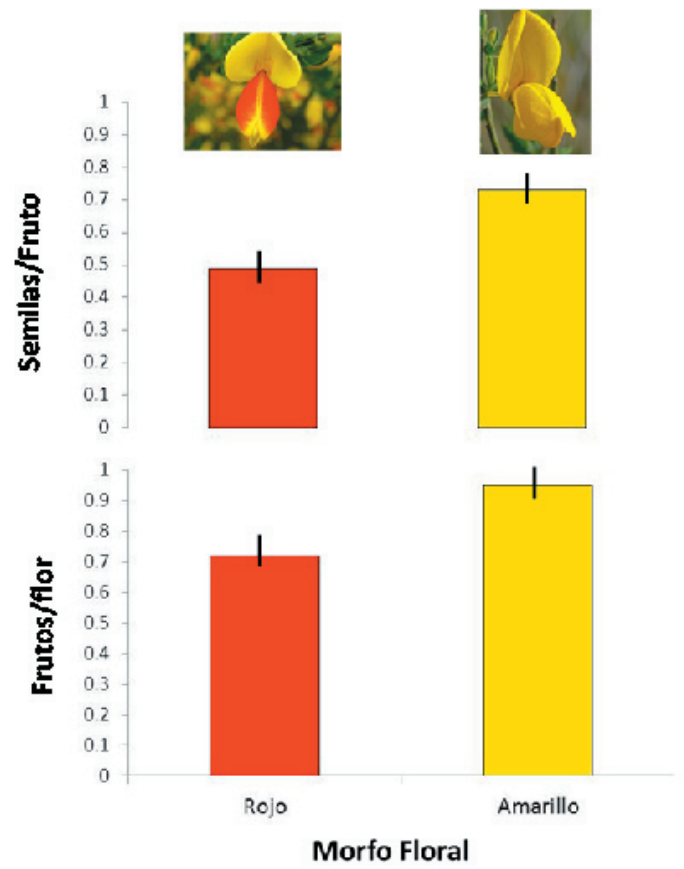

Figura 5. Proporción promedio ( $\pm \mathrm{ES}$ ) de frutos por flor (abajo) y semillas potencialmente viables por fruto (arriba) de individuos de morfos florales amarillos y rojos. Las diferencias entre morfos florales son estadísticamente significativas $(P<0.01$, ver texto).

Figure 5. Mean proportion $( \pm S E)$ of fruit set (i.e., number of fruit per flower, below) and seed set (i.e., number of potentially viable seed per fruit, above) of individuals with red and yellow floral morphs. The differences between floral morphs are statistically significant $(P<0.01$, see text).
Menzel 1992; Skorupski \& Chittka 2010). Los resultados de otras investigaciones, en conjunto con los hallazgos de este trabajo, enfatizan la importancia del color como estímulo que afecta el comportamiento de los visitantes florales. Sin embargo, existen otras alternativas diferentes a cambios en el color de las flores que pueden influir sobre la tasa de visita de los polinizadores eventuales. Primero, la mayor tasa de visita a las flores amarillas puede deberse simplemente a que este morfo floral es más abundante, sin necesidad de proponer la existencia de una preferencia por color. No obstante, en nuestros tratamientos experimentales, las flores amarillas fueron 5 veces más visitadas que las flores rojas o naranjas, pese a que todos los morfos florales presentaban la misma abundancia. En consecuencia, nuestras manipulaciones estarían descartando que la mayor tasa de visita a las flores amarillas se deba a su mayor abundancia, lo que sugiere una preferencia por dicho color. Segundo, la mayor tasa de visita a las flores amarillas puede deberse a una mayor calidad/cantidad de recompensa asociada a este morfo floral. Sin embargo, el hecho que las flores originalmente rojas pintadas de amarillas fueron más visitadas que las rojas (naturales o pintadas) sugiere que el color actúa por sí mismo como una señal atractiva para los visitantes florales, independientemente de la cantidad/calidad de la recompensa. Tercero, las flores de diferentes colores pueden diferir en sus olores o temperaturas y, por ende, la atracción diferencial de los visitantes florales puede deberse más a señales olfativas o térmicas que visuales (Dyer et al. 2006; Dormont et al. 2014). Más allá de que esta hipótesis alternativa necesite de más estudios para ser puesta a prueba, el color seguiría jugado un papel clave, aunque indirecto, en afectar la tasa de visita y la reproducción diferencial de los diferentes morfos florales.

Nuestros resultados sugieren que la mayor abundancia de arbustos con flores amarillas por sobre los que presentan flores rojas o naranjas se debe a una mayor atracción de las flores amarillas por los polinizadores, lo que se traduce en una mayor producción de semillas. Sin embargo, existen otras hipótesis que podrían explicar la mayor abundancia relativa de un morfo floral respecto a otro (Strauss \& Whittall 2006). Es posible que los gradientes ambientales determinen variaciones en el éxito reproductivo de los diferentes morfos, independientemente de 
la tasa de visita. Por ejemplo, la variación latitudinal en la abundancia relativa de las variaciones florales roja y azul de la hierba Lysimachia arvensis se debe a cambios en las condiciones ambientales, que afectan de manera diferencial a la reproducción de estas variantes (Arista et al. 2013). Sin embargo, en el sitio de estudio no es evidente cambios en la abundancia relativa de los morfos de la retama a lo largo de gradientes ambientales; el morfo amarillo es más abundante tanto en el extremo más seco ( $~ 800 \mathrm{~mm}$ de precipitación) como en el más húmedo $(\sim 1600 \mathrm{~mm})$ del gradiente muestreado. Además, los morfos amarillo, rojo y naranja suelen coexistir en un mismo sitio, lo que reduce las posibilidades de que las variaciones en factores ambientales como el clima, fotoperíodo o características de los suelos sean los responsables de sus abundancias diferenciales. Por otra parte, la abundancia relativa de los diferentes morfos florales pueden deberse a limitaciones intrínsecas de cada morfo en los procesos de germinación y establecimiento, idea que necesita de investigaciones adicionales a este trabajo. Finalmente, estamos suponiendo que las variaciones intraespecíficas de los colores de las flores son heredables, condición necesaria para que los visitantes florales funcionen como agentes selectivos. Pese a que no existe información específica sobre la herencia de este carácter en la retama, en general, el color de la flor es heredable (Stanton et al. 1986; Conner 2006). En consecuencia, la hipótesis de que un mayor éxito reproductivo de los arbustos con flores amarillas dejarán como descendencia individuos con flores del mismo color es bastante plausible. Futuros experimentos cruzando polen entre flores de diferentes colores y monitoreando los colores de las flores de las plantas que se producen podrían confirmar esta idea.

Pese a poseer una menor atracción por parte de los visitantes florales y a una menor tasa de reproducción, los arbustos con flores rojas y naranjas, aunque en baja abundancia, se han mantenido presentes en la población a lo largo del tiempo (AGFB, observación personal durante 22 años). Nuestros resultados sugieren que el mantenimiento de estas variantes florales menos visitadas se debe, en parte, a su proximidad a la variante más visitada (i.e., arbustos con flores amarillas). La tasa de visita a arbustos con flores rojas/naranjas decreció de $70 \%$ a $10 \%$ en solo $1 \mathrm{~m}$ (Figura 3 ). Es conocido que la proximidad a un recurso atractivo incrementa la probabilidad de visita/consumo de un recurso menos atractivo (Barbosa et al. 2009). En el ámbito de la polinización, existe gran evidencia de que flores menos atractivas o sin recompensa son más visitadas cuando se encuentran cerca de flores más atractivas o con recompensa (Laverty 1992; Moeller 2004; Molina-Montenegro et al. 2008; Peter \& Johnson 2008). Si este proceso explica el mantenimiento del polimorfismo de color en la retama, nosotros no solo esperamos que la tasa de visita a las flores rojas y naranjas decrezca con su distancia a las flores amarillas (como encontramos), sino que los parches de arbustos con variantes florales rojo y naranja aislados sean raros y poco visitados. Observaciones preliminares confirman estas predicciones.

En resumen, tanto nuestros resultados comparativos como los experimentales sugieren que la mayor abundancia de arbustos de Sarothamnus scoparius con flores amarillas se debe a que este morfo floral es más atractivo para los visitantes florales, lo que se traduce en una mayor tasa de visita y una mayor reproducción comparado con las variantes florales rojo o naranja. Y que la visita a flores rojas y naranjas parece depender fuertemente de la distancia a las flores amarillas. Este trabajo aporta evidencias indirectas sobre la relevancia de los polinizadores como agentes selectivos de las características florales, e ilustra la importancia del contexto (i.e., el vecindario floral) sobre este proceso.

\footnotetext{
Agradecimientos.Este trabajo fue originalmente concebido como el proyecto final de la materia "Ecología de las Interacciones Planta-Animal" de la carrera de Licenciatura en Biología del CRUB-Universidad del Comahue. Agradecemos a todos los que colaboraron en la discusión del mismo y a dos revisores anónimos por sus aportes.
}

\section{BiBLIOGRAFÍA}

Arista, M; M Talavera; R BerJano \& P Ortiz. 2013. Abiotic factors may explain the geographical distribution of flower colour morphs and the maintenance of color polymorphism inthe scarlet pimpernel. J. Ecol., 101:1613-1622.

Barbosa, B; J Hines; I Kaplan; H Martinson; A Szczepaniec; ET AL. 2009. Associational resistance and associational susceptibility: having right or wrong neighbors. Annu. Rev. Ecol. Evol. Syst., 40:1-20.

ChittKa, L \& R Menzel. 1992. The evolutionary adaptation of flower colors and the insect pollinator's color vision. J. Comp. Physiol., 171:171-181.

Conner, JK. 2006. Ecological genetics of floral evolution. Pp. 260-277 en: Harder, LD \& SCH Barrett (eds.). Ecology and Evolution of Flowers. New York: Oxford Univ. Press.

Dyer, AG; HM Whitney; SE Arnold; BJ Glover \& L ChitTKA. 2006. Bees associate warmth with floral color. Nature, 442:525-525. 
Dormont, L; R Delle-Vedove; JM Bessière \& B Schatz. 2014. Floral scent emitted by white and coloured morphs in orchids. Phytochemistry, 100:51-59.

ECKHART, VM; NS RuShing; GM HaRT \& JD HANSEN. 2006. Frequency-dependentpollinator foraging in polymorphic Clarkia xantiana ssp. xantiana populations: implications for flower colour evolution and pollinator interactions. Oikos, 112:412-421.

GALEN, C. 1989. Measuring pollinator-mediated selection on morphometric floral traits: bumblebees and the alpine sky pilot, Polemonium viscosum. Evolution, 43:882-890.

LAnd, M \& L ChittKa. 2013. Vision. Pp. 708-737 en Simpson, SJ \& AE Douglas (eds.). The Insects: Structure and Function, 5th Edition. Cambridge: Cambridge University Press.

LAVERTY, TM. 1992. Plant interaction for pollinator visits: a test for the magnet species effect. Oecologia, 89:502-508.

LEVIN, DA \& ET BRACK. 1995. Natural selection against white petals in Phlox. Evolution, 49:1017-1022.

Meléndez-Ackerman, E \& D CAmpbell. 1998. Adaptive significance of flower color and inter-trait correlations in an Ipomopsis hybrid zone. Evolution, 52:1293-1303

Moeller, DA. 2004. Facilitative interactions among plants via shared pollinators. Ecology, 85:3289-3301.

Molina-Montenegro, M; E Badano \& L CAVIeres. 2008. Positive interactions among plant species for pollinator service: assessing the 'magnet species' concept with invasive species. Oikos, 117:1833-1839.

Muller, H. 1883. The fertilization of owers. MacMillan and Co. London.

PapioreK, S; K Rohde \& K Lunau. 2013. Bees' subtle colour preferences: how bees respond tosmall changes in pigment concentration. Naturwissenschaften, 100: 633-643.
Peter, C \& S Johnson. 2008. Mimics and magnets; the importance of color and ecological facilitation in floral deception. Ecology, 89:1583-1595.

Renoult, J; A VAlido; P Jordano \& HM Schaefer. 2014: Adaptations of flower and fruit colours to diversified mutualists. New Phytologist, 201:678-686.

Rodríguez-Riaño, T; A Ortega-Olivencia \& J Devesa. 1999. Reproductive biology in two Genisteae (Papilionoideae) endemic of the western Mediterranean region: Cytisus striatus and Retama sphaerocarpa. Can. J. Bot., 77:809820.

SkORUPSKI, P \& L ChitTKa. 2010. Photoreceptor Spectral Sensitivity in the Bumblebee, Bombus impatiens (Hymenoptera: Apidae). PLos One, 5:e12049.

STANTON, ML. 1987. Reproductive biology of petal color variants in wild populations of Raphanus sativus. I. Pollinator response to color morphs. Am. J. Bot., 74: 178-187.

Stanton, ML; AA SnOW \& SN Handel. 1986. Floral evolution: attractiveness to pollinators increases male thess. Science, 232:1625-7.

STrauss, SY \& JB WhitTaLl. 2006. Non-pollinators agents of selection on floraltraits. Pp. 120-138 en: Harder, LD \& SC Barrett (eds.). Ecology and Evolution of Flowers. Oxford University Press, New York, NY, USA.

STREISFELD, MA \& JR KoHN. 2007. Environment and pollinator-mediated selection on parapatric floral races of Mimulus aurantiacus. J. Evol. Biol., 20:122-132.

WAser, NM \& MV PrICE. 1981. Pollinator choice and stabilizing selection for flower color in Delphinium nelsonii. Evolution, 3:376-390.

WeISS, MR. 1995. Floral color change: a widespread functional convergence. Am. J. Bot., 82:167-185. 